\title{
La influencia térmica de la pared de lodo del espacio solar en el clima interior de la casa de Valparaíso 1811 - 1950
} PABLO CASTAÑER JERIA

\author{
$>$ Arquitecto. Escuela de Arquitectura Universidad de Valparaíso, Chile \\ pablo.castaner@uv.cl \\ ORCID 0000-0002-8760-4047 \\ NINA HORMAZABAL POBLETE \\ $>$ Arquitecta. Escuela de Arquitectura Universidad Federico Santa María, Chile \\ nina.hormazabal@usm.cl \\ ORCID 0000-0002-7607-7675 \\ GERARDO SAELZER FUICA \\ $>$ Arquitecto. Universidad del Bio-Bío, Chile \\ gsaelzer@ubiobio.cl \\ ORCID 0000-0002-4289-8379
}

Universidad de Valparaíso Facultad de Arquitectura Revista Márgenes Espacio Arte Sociedad La influencia térmica de la pared de lodo del espacio solar en el clima interior de la casa de Valparaíso 1811 - 1950

Diciembre 2021 Vol $14 \mathrm{~N}^{\circ} 21$

Páginas 91 a 100

ISSN electrónico 0719-4436

Recepción abril 2021

Aceptación julio 2021

DOI 10.22370/margenes.

2021.14.21.3104

\section{RESUMEN}

Este estudio descriptivo exploratorio analiza el aporte del adobillo al espacio solar de las casas de Valparaíso construidas durante la $4^{\text {ta }}$ etapa de crecimiento de la ciudad de Valparaíso, período de 1811 a la década de 1950. Los espacios solares formaron parte de los sistemas Globo y Marco de Plataforma, cuyas paredes de masa se rellenaron con adobillo, esta característica se analiza desde un aspecto técnico para estudiar su influencia en el confort térmico.

Para estudiar el comportamiento térmico del espacio solar y el muro de adobillo se aplicaron dos herramientas de medición, dataloggers y simulación térmica dinámica, que ayudan a identificar los factores que contribuyen a la eficiencia energética, y especialmente, cómo contribuyen el espacio solar y el muro de adobillo al clima interior de estas casas del patrimonio histórico de Valparaíso.

Primero, para entender el espacio solar, "galería” en español, se realiza un análisis histórico en la ciudad de Valparaíso, identificando el componente arquitectónico ambiental y sus partes, reconociendo los valores patrimoniales presentes en la ciudad como resultado del aporte arquitectónico de migrantes ingleses y estadounidenses que trajeron las técnicas constructivas. En segundo lugar, se mide y simula este espacio y sus elementos, se analizan y contrastan los datos obtenidos con ambas herramientas.

Los resultados corroboraron que el Marco de Globo relleno con muro de adobillo es responsable del control térmico de los espacios adyacentes, actuando como mediador entre estos dos ambientes, donde el espacio solar es a su vez el mediador del ambiente externo y el ambiente interior. Determinar que este componente arquitectónico ambiental pasivo contribuye favorablemente al confort térmico en el espacio solar y en el hogar en su conjunto. Finalmente, las conclusiones obtenidas podrían potencialmente servir para la futura rehabilitación energética de viviendas patrimoniales en Valparaíso y/o posibles nuevas aplicaciones de este componente ambiental.

PALABRAS CLAVE

casas patrimoniales de Valparaíso, espacio solar, rendimiento térmico

The thermal influence of the mud wall of the sunspace in the indoor climate of the 1811 - 1950 Valparaíso house ABSTRACT

This exploratory descriptive study analyses the contribution of the mud wall, "adobillo" in Spanish, of the sunspace of the houses of Valparaiso built during the $4^{\text {th }}$ stage of growth of the city of Valparaiso, period from 1811 to 1950's. The sunspaces were part of the Balloon and Platform Frame systems, which mass walls were filled with adobillo, this feature is analyzed from a technical aspect to study its influence on the thermal comfort.

To study the thermal performance of the sunspace and the adobillo wall, two measurement tools were applied, data-loggers and thermal dynamic simulation, which help to identify the factors that 
contribute to energy efficiency, and especially, how the sunspace and the adobillo wall contribute to the indoor climate of these houses of the historical heritage of Valparaíso.

First, to understand the sunspace, "galería" in Spanish, a historical analysis is carried out in the city of Valparaíso, identifying the environmental architectural component and its parts, recognizing the heritage values present in the city as a result of the architectural contribution of English and American migrants that brought the constructive techniques. Second, this space and its elements are measured and simulated, the data obtained with both tools was analyzed and contrasted.

The results corroborated that the Balloon Frame filled in with adobillo wall is responsible for the thermal control of the spaces adjacent to it, acting as a mediator between these two environments, where the sunspace is in turn the mediator of the external environment and the indoor environment. Determining that this passive environmental architectural component favorably contributes to thermal comfort in the sunspace and in the home as a whole. Finally, the conclusions obtained could potentially serve for future energy rehabilitation of patrimonial housing in Valparaíso and/or possible new applications of this environmental component.

KEYWORDS

heritage houses of Valparaíso, sunspace, thermal performance

\section{INTRODUCCIÓN}

Valparaíso se caracteriza por ser una de las ciudades más visitadas del país producto de su nombramiento en 2003 , como patrimonio de la humanidad por la UNESCO, su importancia histórica radica en que es considerada un testimonio de los inicios de la fase temprana de la globalización comercial y cultural a fines del siglo XIX, un aspecto importante en su nombramiento, es su valor arquitectónico histórico producto de las migraciones (UNESCO - ICOMOS, 2014), donde destacan las galerías vidriadas de las viviendas, las cuales poseen características ambientales pasivas, solamente por su conformación constructiva aportando al confort térmico de los habitantes.

Actualmente, se evidencia un deterioro paulatino de esta arquitectura histórica en Valparaíso (Figura 1), y particularmente de las galerías vidriadas, las cuales sufren por fenómenos destructivos como incendios, plagas de termitas, terremotos, especulación inmobiliaria, falta de mantención entre otros y una nula normativa que las proteja. Otro aspecto es el escaso conocimiento de su comportamiento y conformación, por la carencia de estudios, mediciones y levantamientos de datos preliminares que permitan la toma de decisiones arquitectónicas para la mantención, intervención y rehabilitación de estos componentes de arquitectura pasiva. Resulta entonces atractivo poder estudiar estos componentes holísticamente, considerando herramientas de investigación cualitativas y cuantitativas.

El presente estudio descriptivo exploratorio analiza la contribución del muro de barro, adobillo, que se encuentra presente en las galerías vidriadas de las casas de Valparaíso construidas durante la cuarta etapa de crecimiento de la ciudad de Valparaíso, período entre 1811 a 1950 aproximadamente. Las galerías vidriadas eran un espacio de la arquitectura residencial utilizado en los sistemas anglosajones conocidos como Balloon Frame y Platform Frame, cuyas paredes masivas se caracterizaban por estar compuestas por una estructura portante de madera rellena de adobillo, esta característica se analiza desde un aspecto técnico para estudiar su influencia en el confort térmico. El adobillo refiere a una técnica constructiva mixta compuesta por barro y madera, que en el caso de Valparaíso la estructura portante se conformaba por un entramado de madera que se rellenaba con ladrillos de tierra, donde estos últimos no tenían ningún compromiso estructural (CDT, 2012).

\section{HISTORIA Y CONTEXTO DE LAS GALERÍAS VIDRIADAS DE VALPARAÍSO}

El origen de la galería vidriada europea en inglés conservatory o sunspace, surge de una adaptación de los invernaderos del siglo XIX a la arquitectura residencial aprovechando sus cualidades climáticas y espaciales, es por ello, que ha sido estudiada por diversos investigadores con distintos enfoques, ya sea por: la relación de superficies translúcidas y opacas, iluminación natural, demanda y consumo energético, tipo de envolvente, tipos de vidrios y la relación de la masa térmica en el aporte al confort térmico en la galería vidriada. Para la presente investigación la característica distintiva principal de la galería vidriada de Valparaíso, es que posee un muro Balloon Frame o Platform frame relleno de adobillo que comunica la galería con el interior de la vivienda.

La historia de la arquitectura nos indica que, los invernaderos, elementos de donde provienen las galerías, nacen en el siglo XVIII, producto de la evolución y el desarrollo en las ciencias de los materiales (Kostof, 2003). Estas construcciones si bien, se encontraban aisladas en los jardines, con el paso del tiempo se comenzaron a adosar a las fachadas posteriores o laterales como espacios de transición entre la arquitectura y el jardín, como fue corriente en las villas suburbanas y en los palacetes de la nobleza y las aristocracias de las grandes capitales europeas. Fueron tan populares entre la aristocracia y la nobleza, que se prefabricaron en finos materiales para cerrar balcones o adosar a fachadas iluminadas por el sol (Rodríguez, 2001).

Rodríguez señala que a finales de siglo XIX, Balbino Cortés realiza uno de los primeros estudios exhaustivos de los invernaderos y su construcción en la Novísima guía de hortelano, jardinero y arbolista (Cfr. Cortés, 1885), donde dedica todo un capítulo a los invernáculos y estufas (sunspace). Este jardinero explica que, aunque el hierro es más elegante y duradero, la estufa (nombre que da España a la galería vidriada) construida de madera tiene la ventaja de aumentar y disminuir su temperatura de forma paulatina en el tiempo, además de no concentrar una cantidad de vapor en su interior debido a la naturaleza del comportamiento de la madera. También llegó a establecer tres tipos de invernáculos; los fríos, los templados y los calendarios $u$ holandeses, donde cada uno maneja distintos grados de temperaturas, las primeras entre $4^{\circ} \mathrm{C}$ y $5^{\circ} \mathrm{C}$, 


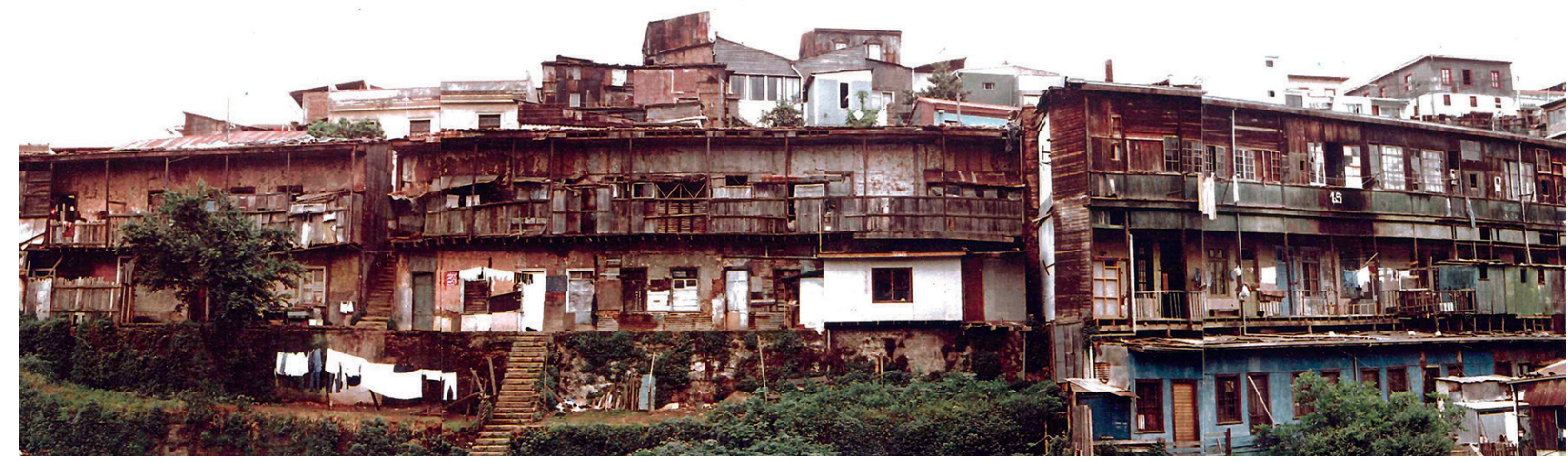

las segundas entre $8^{\circ} \mathrm{C}$ y $10^{\circ} \mathrm{C}$ y finalmente entre $15^{\circ} \mathrm{C}$ y $30^{\circ} \mathrm{C}$ (Rodríguez, 2001).

Los invernaderos proliferaron a mediados del siglo XIX, cuando estos materiales bajaron sus costos, fenómeno que se extendió a los pequeños palacios y casas con modelos industrialmente prefabricados para adherir a las fachadas y techos (Rodríguez, 2001). Es justo en este periodo cuando el invernadero comienza a lograr otro protagonismo como elemento social y ambiental incorporándose a la arquitectura habitacional del siglo XIX, pues además de ser un lugar de aclimatación de plantas y especies exóticas se convierte en un lugar de fiestas y reuniones sociales de los aristócratas y nobles de la época, socialmente atrayente como símbolo de estatus y distinción en Europa (Figura 3), tal como Simó los describe en la revista Fragmentos en su artículo Formación del espacio burgués (Simó, 1989).

\section{ANTECEDENTES}

\section{La galería vidriada}

Estos nuevos preceptos de la arquitectura comienzan a expandirse por todo el mundo a través de los puertos; el puerto además de ser la puerta de entrada y salida de la población (Harris, 1996), fue el principal canal de intercambio comercial y cultural mediante las actividades económicas, mercantilismo, actividades portuarias y flujo de inmigrantes en busca de oportunidades y/o establecimiento de negocios, lo que hace de Valparaíso una ciudad cúspide para el asentamiento de los mismos y surgimiento de la cultura portuaria y multicultural, pero no solo a nivel de ciudad, sino que también a nivel del país y la región. Por el año 1831, el puerto de Valparaíso ocupa un lugar privilegiado dentro de las costas del Pacífico, y entre 1810 y 1837 se contabilizan más de 7.000 naves arribadas en el puerto (Estrada, 1978), de este modo Valparaíso llegó a posicionarse como principal puerto del Pacífico Sur atrayendo a miles de inmigrantes de todos los estratos socioeconómicos, europeos y norteamericanos que llegan a Chile, trayendo y marcando con su cultura e influencia el desarrollo de la ciudad, esta influencia, entre muchos otros aspectos otorgaba valor a la función del espacio público, del ocio y las nuevas tecnologías constructivas (Estrada, 1978). Es así entonces, como el sistema constructivo Balloon Frame, Platform Frame y sus derivados y las galerías vidriadas proliferan en las nuevas construcciones residenciales realizadas por los inmigrantes que venían a instalarse a Valparaíso, donde además pareciera que la intuición del inmigrante y las condiciones topográficas y climáticas propiciaron la implantación y adecuación de sistemas y modas arquitectónicas de la época, resultando en sistemas y componentes muy apropiados a su entorno (Figura 4).
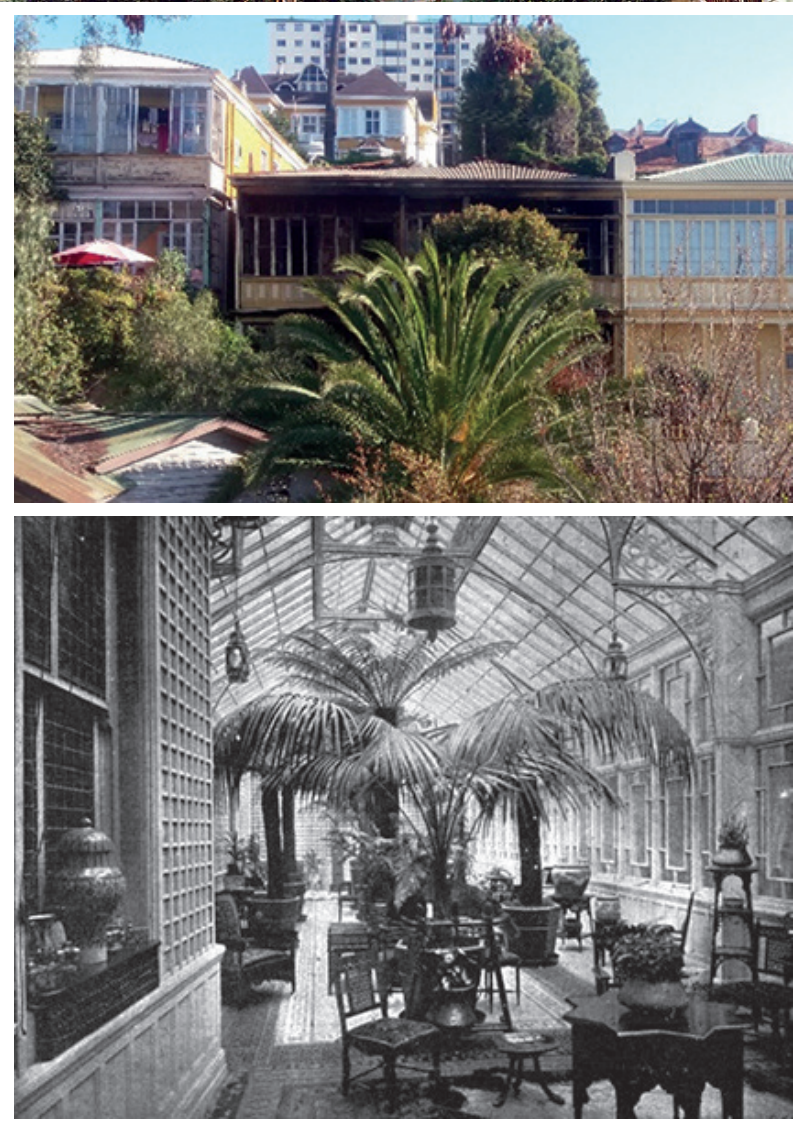

$>$ Figura 1. Fotografía del deterioro evidente de una típica ladera de uno de los cerros de Valparaíso con algunas galerías vidriadas. Registro del autor, 2018 .

$>$ Figura 2. Fotografía de típicas galerías vidriadas de Valparaíso, Cerro Alegre. Registro del autor, 2018.

$>$ Figura 3. Fotografía de invernadero como salón social. Fuente: www. victorianweb.org 

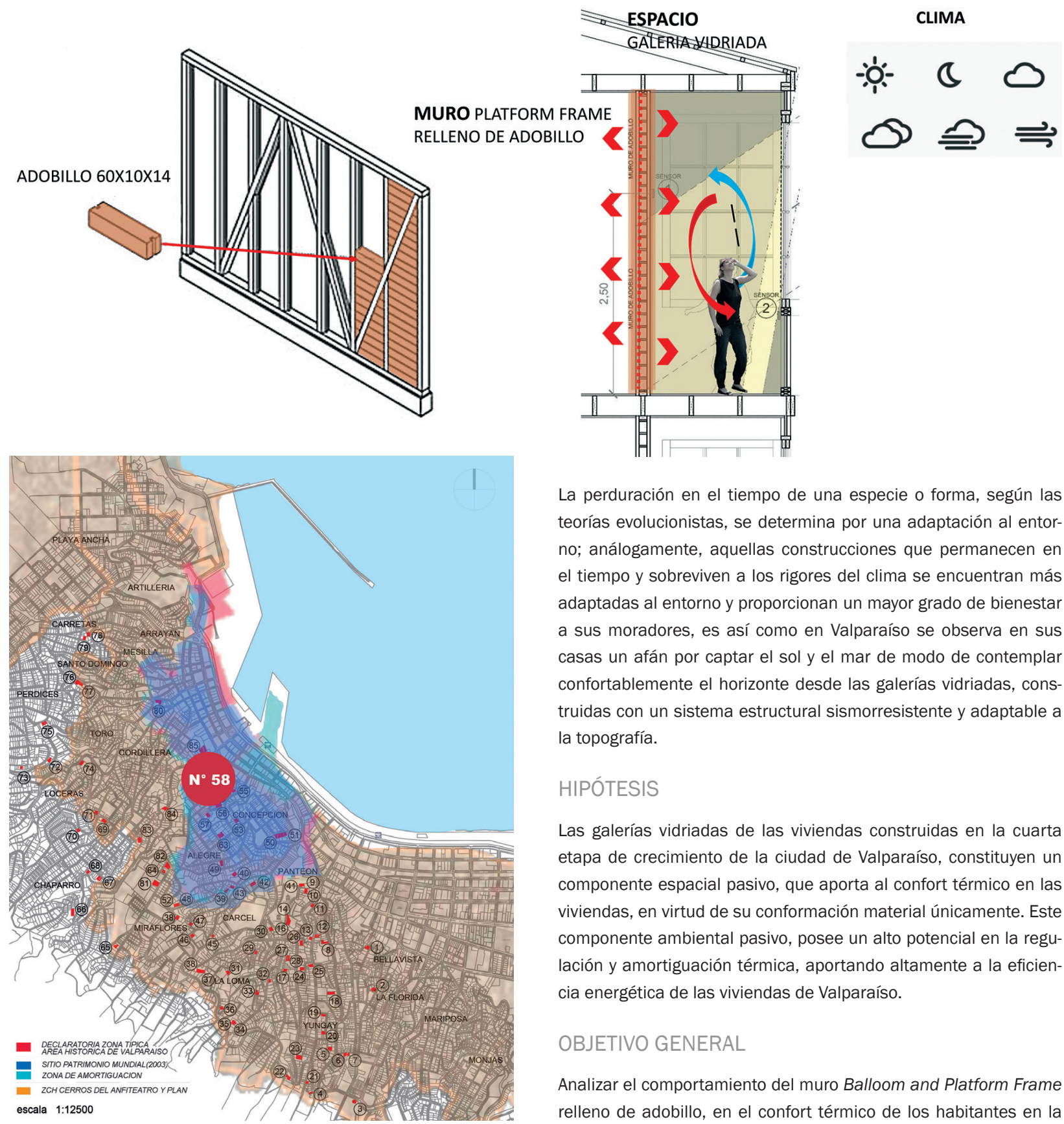

La perduración en el tiempo de una especie o forma, según las teorías evolucionistas, se determina por una adaptación al entorno; análogamente, aquellas construcciones que permanecen en el tiempo y sobreviven a los rigores del clima se encuentran más adaptadas al entorno y proporcionan un mayor grado de bienestar a sus moradores, es así como en Valparaíso se observa en sus casas un afán por captar el sol y el mar de modo de contemplar confortablemente el horizonte desde las galerías vidriadas, construidas con un sistema estructural sismorresistente y adaptable a la topografía.

\section{HIPÓTESIS}

Las galerías vidriadas de las viviendas construidas en la cuarta etapa de crecimiento de la ciudad de Valparaíso, constituyen un componente espacial pasivo, que aporta al confort térmico en las viviendas, en virtud de su conformación material únicamente. Este componente ambiental pasivo, posee un alto potencial en la regulación y amortiguación térmica, aportando altamente a la eficiencia energética de las viviendas de Valparaíso.

\section{OBJETIVO GENERAL}

Analizar el comportamiento del muro Balloom and Platform Frame relleno de adobillo, en el confort térmico de los habitantes en la galería vidriada de Valparaíso, desde el punto de vista de la masa térmica, captación solar y pérdidas térmicas a través de su orientación, conformación geométrica y material únicamente.

Para comprobarlos se estableció una metodología mixta, cualitativa y cuantitativa, típicamente utilizada en investigación arquitectónica de este tipo, cuyas actividades e instrumentos metodológicos se dividieron en dos grupos. Primero, para comprender el espacio de la galería vidriada, se realiza un análisis histórico en la ciudad de Valparaíso, identificando, catastrando y relevando detalladamente el componente arquitectónico ambiental de galerías vidriadas (Figura 2), y sus partes, reconociendo los valores patrimoniales de éstas presentes en la ciudad como resultado de la contribución arquitectónica de migrantes ingleses y estadounidenses que trajeron las técnicas constructivas cuando se instalaron a vivir en Valparaíso. Segundo, este espacio y sus elementos fueron medidos y simulados con sensores ambientales y simulaciones computacionales, cuyos datos obtenidos con ambas herramientas fueron analizados, contrastados y validados entre sí. El objetivo de este 

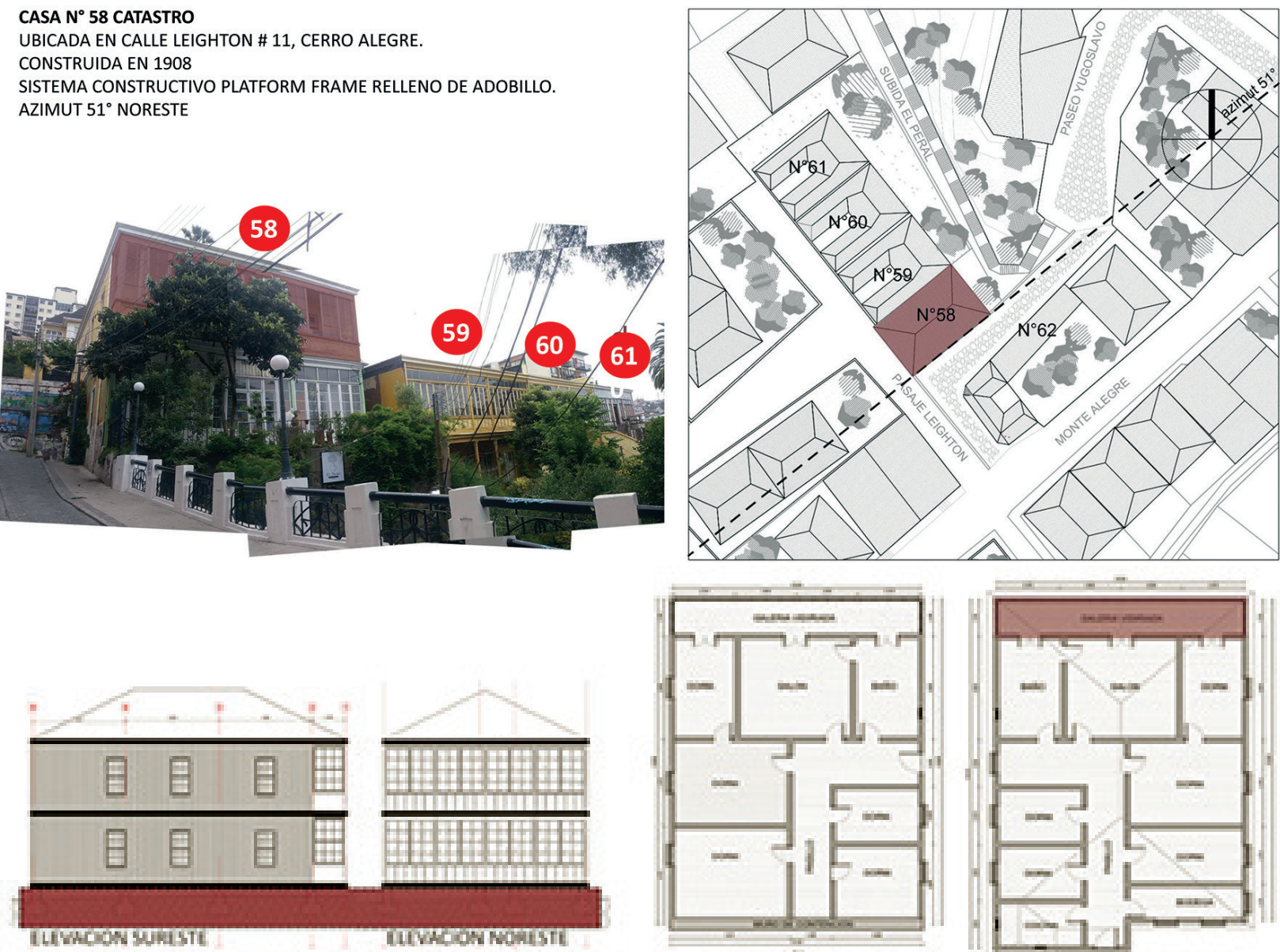

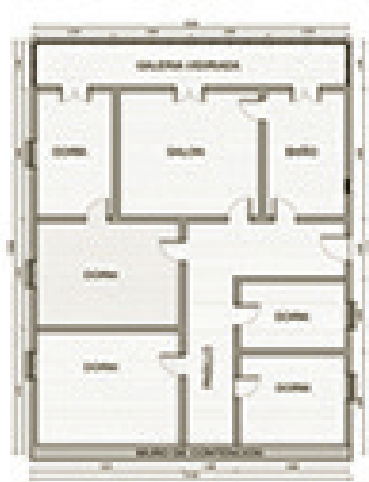

Planta nives

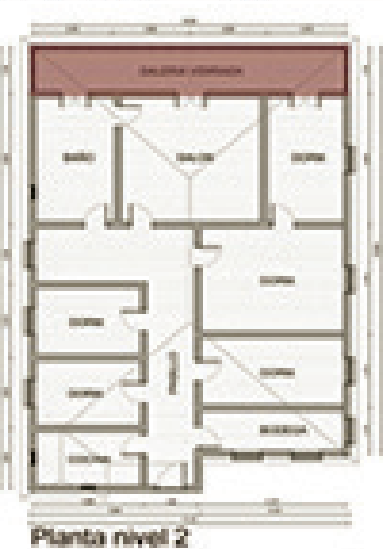

segundo grupo de actividades y aplicación de instrumentación pertinentes fue estudiar el desempeño térmico de la galería vidriada y la pared de adobillo. Las herramientas de medición se aplicó con medidores (dataloggers) instalados in situ para medir temperaturas de aire y superficiales y humedad relativa del aire, a distintas alturas, y la herramienta de simulación dinámica computacional se realizó con el software DesignBuilder $\AA^{\circledR}$, ambas con el objeto de identificar los factores que contribuyen a la eficiencia energética y, especialmente, comprender la influencia de la galería vidriada y el muro de adobillo en el clima interior de estas casas del patrimonio histórico de Valparaíso.

\section{RESULTADOS}

\section{Caso de Estudio, una vivienda en Platform Frame con muro de adobillo}

A continuación, se realizará la descripción del caso de estudio mediante planimetría arquitectónica, mediciones in situ y simulaciones dinámicas, se realizará un análisis del desempeño térmico ambiental de la galería vidriada y de la influencia del muro Platform Frame con relleno de adobillo, con respecto a las horas de confort según modelo de confort térmico pre-establecido.

\section{Zona de Catastro}

Se elabora un catastro demostrativo de los casos de viviendas construidas con sistema Balloon y Platform Frame y sus derivados con galería vidriada, considerando: materialidad, detalles constructivos, estilo arquitectónico, proporciones geométricas, se logró identificar y catastrar 85 casos visualmente detectables (Figura 5), considerando que debido a la gran superficie del estudio y las

$>$ Figuras 6 y 7. Izquierda: Fotografía fachada Calle Leighton en Cerro Alegre. Derecha: Plano de ubicación Caso de Estudio № 58. Elaboración propia.

$>$ Figura 8. Planimetría vivienda Caso de Estudio № 58. Izquierda a derecha: Elevaciones Sureste y Noreste, Plantas Niveles 1 y 2 . Elaboración propia 


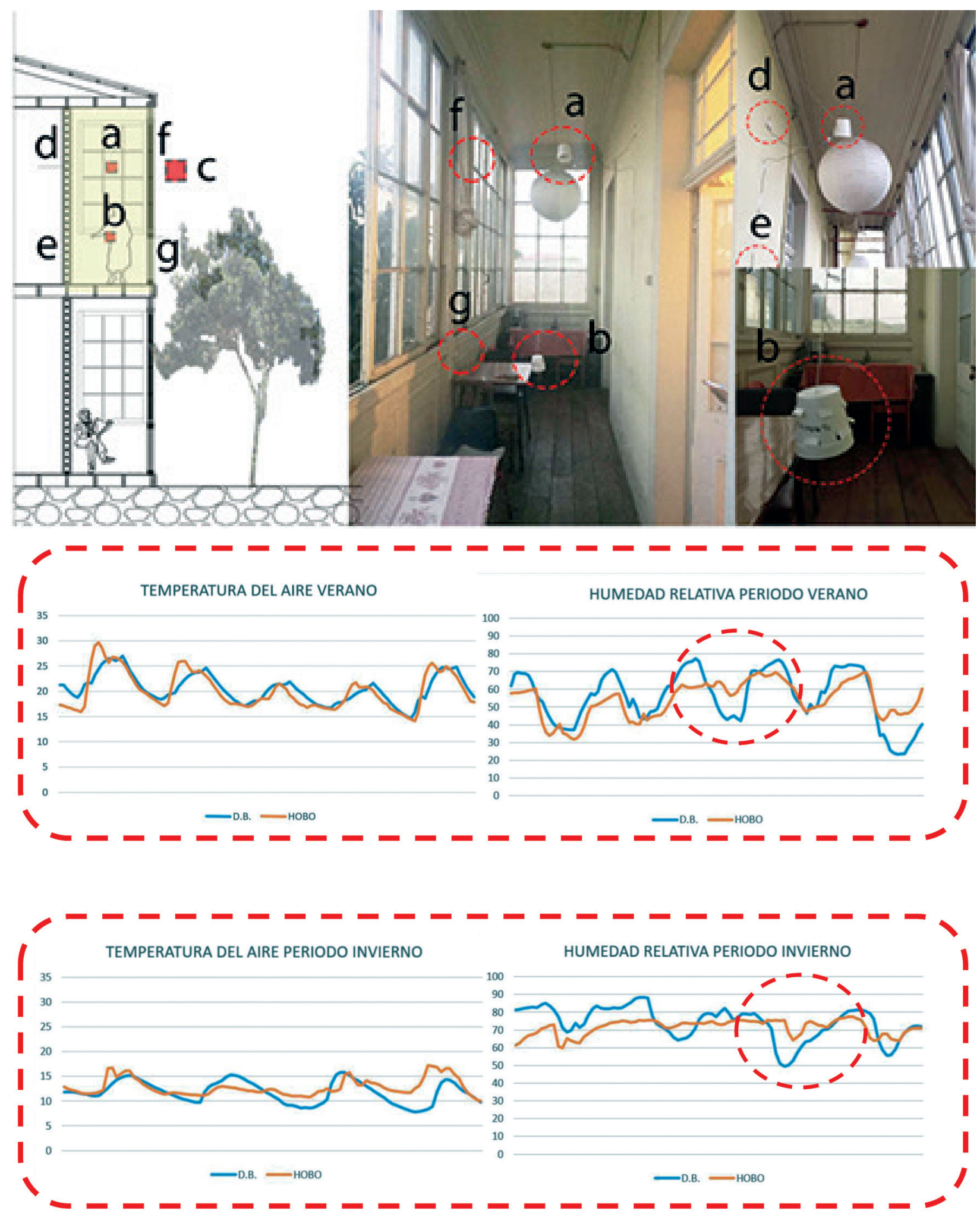

$>$ Figura 9. Localización de sensores periodo febrero 2019, sección y

fotomontaje con dataloggers: a) y b) To aire interior 2.5 y $1.0 \mathrm{~m}$ altura; c) $\mathrm{T}^{\circ}$

aire exterior; d) y e) TC To superficie $2.5 \mathrm{~m}$ y $1.0 \mathrm{~m}$ altura muro adobillo; f) y g)

TC To superficie 2.5 y $1.0 \mathrm{~m}$ altura marco madera. Elaboración propia.

$>$ Figura 10. Gráficos de datos obtenidos con dataloggers ( $\mathrm{HOBO} 囚)$ contribuyen en validar los datos obtenidos con las simulaciones (DesignBuilder®). Elaboración propia. 
condiciones topográficas de la ciudad se sabe que existen muchos más, los que no se ven a simple vista debido a que sus fachadas han quedado ocultas debido a la expansión de la ciudad.

\section{Caso de Estudio}

La dificultad de conseguir autorización de los propietarios para estudiar y tomar mediciones in situ en su vivienda nos llevó a elegir un caso de estudio representativo y conocido en Valparaíso. El sector donde se ubica el caso de estudio es en la parte más alta de la escalera y ascensor El Peral en calle Leighton N¹1, Cerro Alegre, Valparaíso. En la pequeña meseta sobre el muelle Prat y la plaza Sotomayor. Indicado con el $N^{\circ} 58$ en el plano de ubicación e imágenes del caso de estudio a continuación, incluidas en figuras 6,7 y 8 .

\section{Mediciones}

Los instrumentos fueron instalados (Figura 9) dentro de la galería vidriada a modo de medir las variables de humedad relativa, temperatura interior, temperatura del muro interior y temperatura exterior, estos fueron instalados según protocolo de mediciones de espacios interiores y con el objetivo de comprender el desempeño higrotérmico de la galería vidriada y la influencia del muro de adobillo en comparación y complementación con la simulación dinámica y así obtener cruce de los datos obtenidos. Las mediciones se realizaron en agosto y diciembre de 2018 y febrero 2019 respectivamente.

\section{Simulaciones}

La simulación dinámica computacional consistió en una combinación de mediciones empíricas, verificaciones analíticas y técnicas de análisis comparativos. Se comenzó con el diagnóstico y verificación analítica con mediciones in situ con equipos calibrados y estandarizados, luego, obtención y recolección de datos experimentales, que se incorporaron al archivo de entrada del programa de simulación dinámica, posteriormente, del archivo de salida se obtiene información valiosa para el caso de estudio, ya que las variables utilizadas fueron medidas experimentalmente, con ello robustece y valida el modelo (Figura 10), obteniéndose una situación panorámica muy cercana a la realidad en cuanto a las horas de confort. Algunas de las simulaciones realizadas y presentadas a continuación (Figura 11) se basan en variables parametrizadas debido a la factibilidad del uso de ciertos equipos, con ello, se obtuvo una idealización de la realidad y las horas de confort.

En primer lugar, con lo anterior se reconoce cómo y cuáles variables influyeron en el rango de confort estudiado para luego optimizar es-
$>$ Figura 11. Matriz de simulaciones realizadas bajo las variables establecidas. Elaboración propia.

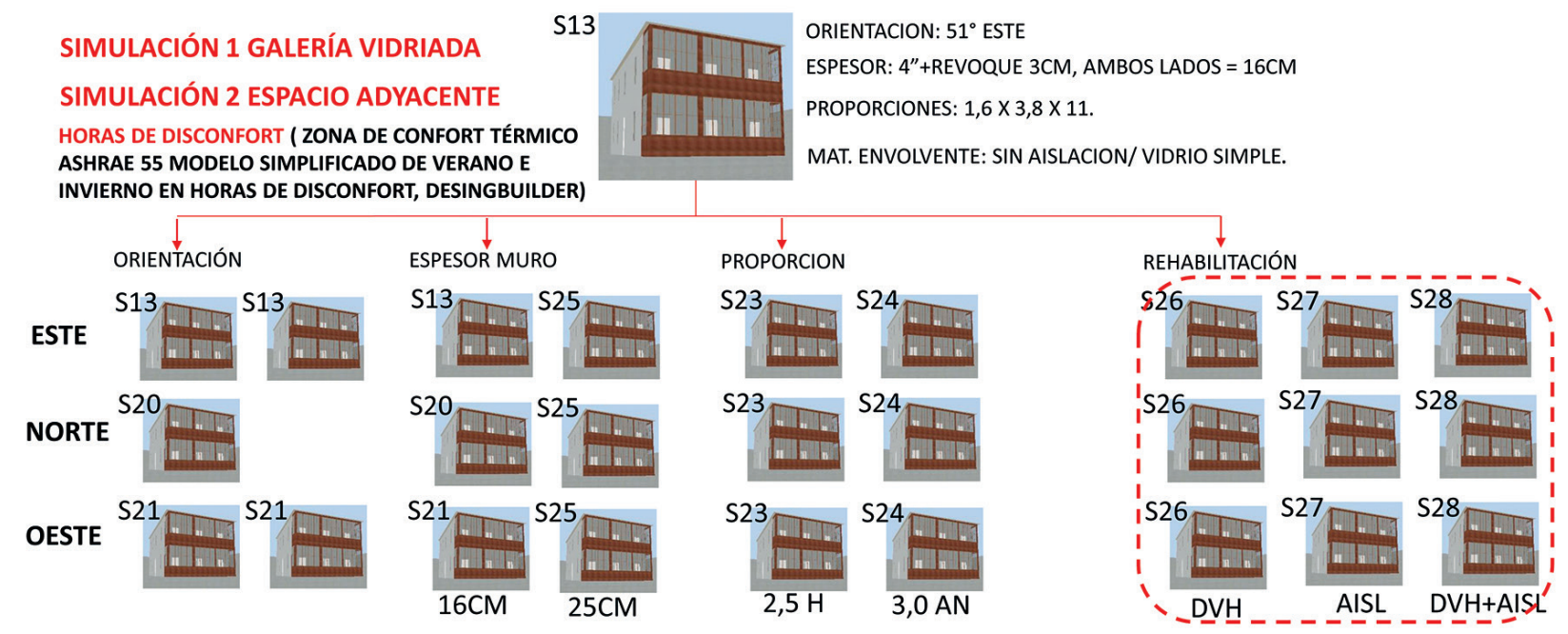


Tabla 2. Resumen de resultados obtenidos del desempeño térmico de la galería vidriada. tas variables para un rango de confort aceptable. Para obtener los aportes al confort térmico del sistema constructivo, se consideró la influencia del espesor, la orientación óptima y las dimensiones de la galería vidriada, al modificar estos aspectos en cada nueva simulación se pretendía alcanzar un rango de confort óptimo.

Cabe mencionar, que las simulaciones posteriores (últimas cuatro columnas de la derecha) donde se incluyeron datos de infiltración, ventilación (CITEC, 2014) y conductividad térmica de muros y vidrios, se realizaron considerando los cálculos establecidos en los estándares nacionales (MINVU, 2018), a continuación, se muestra tabla 1 comparativa de los cálculos manuales de valor $U$ realizados según la NCh 853 (INN, 2007) en contraste con los obtenidos a través de la simulación (S13 base) con el software DesignBuilder®.

> Tabla 1. Comparación cálculo de transmitancias térmicas muro adobillo.

Sim. 13 Transmitancia térmica Valor $\mathrm{U}\left(\mathrm{W} / \mathrm{m}^{2} \mathrm{~K}\right)$

\begin{tabular}{rrrr}
\hline m. exterior & m. interior & \multicolumn{1}{c}{$\begin{array}{c}\text { vidrio } \\
\text { simple }\end{array}$} & \multicolumn{2}{c}{ m. madera } \\
\hline 2,893 & 2,626 & 5,8 & 3,2 \\
\hline 2,23 & 2,38 & 5,8 & 2,94
\end{tabular}

Cálculo

Nch 853

Simulaciones térmicas

La Tabla 2 a continuación presenta los resultados más relevantes de las distintas simulaciones dinámicas respecto del confort térmico considerando los aspectos de orientación (S3, S20, S21), aumento de espesor (S22, S22 N, S22 0), proporciones (S23, S24, S23 N, S23 0) y mejoras para la rehabilitación (S26, S27, S28, S29).

Según Tabla 2 en cuanto a las horas de confort se modificó el espesor del muro en estudio, para S22 al aumentar los espesores de las capas de revoque y espesor del adobillo, se obtuvo el mejor desempeño en la orientación este, con notorias disminuciones de las horas de disconfort en los períodos de verano y primavera. Las simulaciones S22 N y S22 O registran mejoras con respecto al S13 en los periodos de otoño e invierno debido a factor de la orientación, sin embargo, para los períodos más cálidos se observa sobrecalentamiento producto del aumento de las temperaturas y baja en la humedad relativa atribuibles a la orientación poniente ya que el sol al estar más horizontal y la galería al tener 3.8 metros de altura capta mayor cantidad de ganancias solares. Este fenómeno

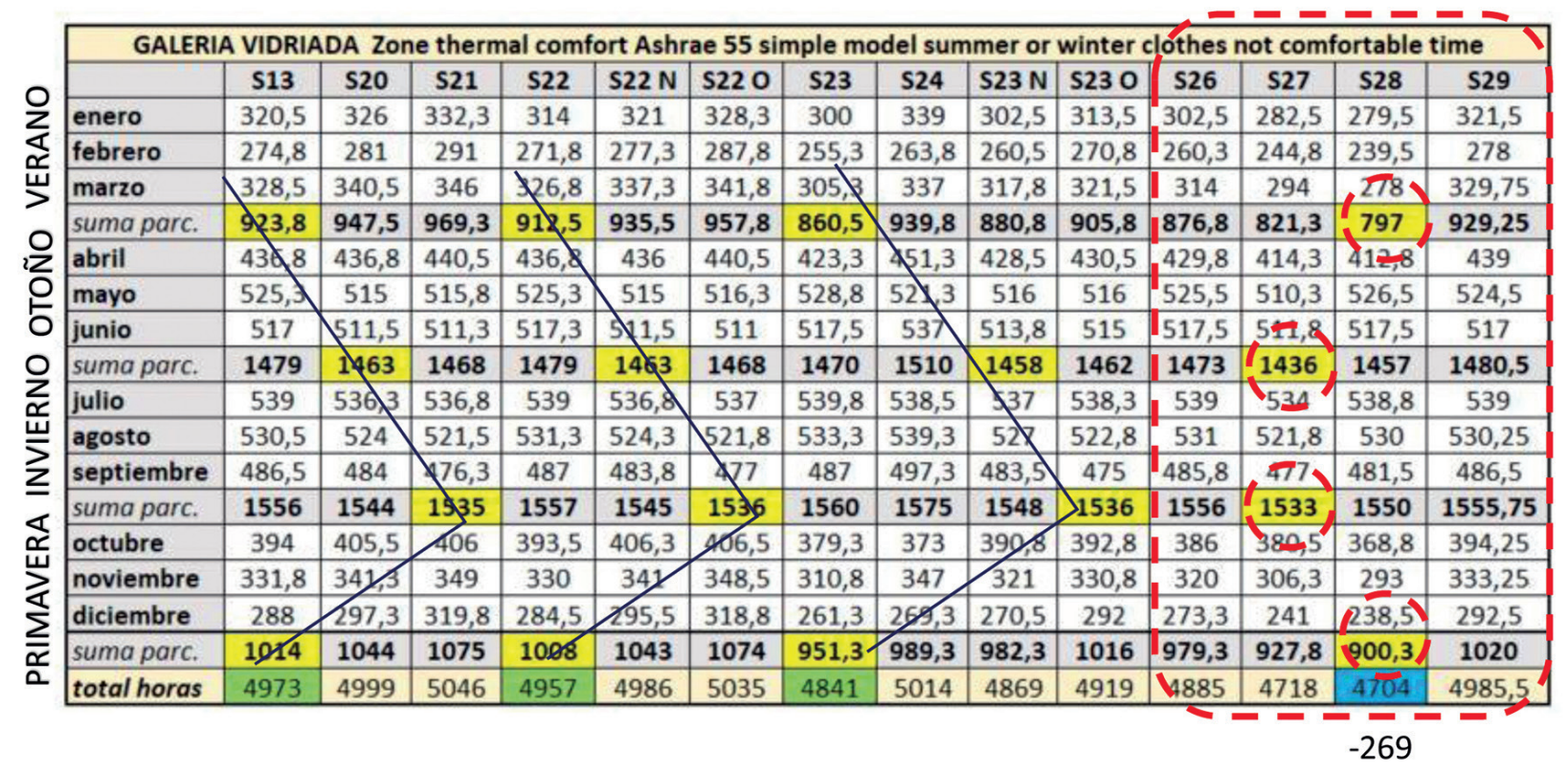


se ve reflejado en un doble pick de las curvas del sensor colocado a la altura de 2.5 metros en períodos calurosos, puesto que se observa que las proporciones del sistema constructivo Balloon y Platform Frame, debido a su altura una mayor cantidad de superficie vidriada de captación solar, fenómeno que se ve favorecido en las orientaciones oriente y poniente (doble pick), de acuerdo al registro de las simulaciones se concluye que se debe requerir de estrategias de diseño para prevenir el sobrecalentamiento en caso de orientación poniente.

La disminución de la altura de 3.8 metros a 2.5 metros reduce las horas de disconfort en un $1.4 \%$, en la sumatoria anual para la orientación oriente, los que se dan en primavera y verano, indicando que al tener menos altura se tiene menos superficie de captación solar para los meses de otoño e invierno donde el ángulo del sol es más bajo. Esta simulación se lleva a cabo en orientación norte (S23 N) y poniente (S23 0) obteniendo similares resultados.

Por otra parte al simular una galería vidriada más ancha, lo resultados muestran que no hay reducción en las horas de disconfort, ya que aumenta en demasía el volumen de aire y el muro se distancia demasiado de la superficie vidriada perdiendo eficacia para la captación solar, por lo anterior se puede establecer que las proporciones de la galería vidriada en su ancho y alto, producto del sistema constructivo estudiado ayudan en el confort térmico de la galería vidriada, obteniendo mayor superficie de captación solar a través de la masa térmica en el muro, para los mismos metros cuadrados, en los periodos más fríos.

Respecto a la influencia de la galería vidriada y el muro Platform Frame relleno de adobillo en la zona adyacente interior (Zona 2) las simulaciones anuales arrojan que para la orientación poniente se reducen las horas de disconfort, siendo la acción combinada de aumento de espesor de muro y orientación poniente (S22 Oeste) la que obtiene mejores resultados comparado con la S13, para la zona adyacente. También en los resultados se observa que para el periodo de mayo a agosto se obtienen un total de horas de disconfort sin alteraciones donde ninguna de las simulaciones pudo mejorar los índices, por lo cual para estos meses se requiere de estrategias activas para disminuir las horas de disconfort y mejorar el confort térmico. También se puede agregar que las simulaciones con orientación norte ayudan a disminuir las horas de disconfort en invierno y otoño para los meses de abril y septiembre al igual que para la galería vidriada.

El análisis de las mejoras de rehabilitación nos muestra que en general las dos estrategias por separado y confinadas disminuyen las horas de disconfort, la estrategia de menor impacto es el cambio a ventanas DVH que disminuye un 1.8\% en la comparación anual, donde las mejoras se concentran en los periodos de verano y primavera a diferencia de la estrategia de aislación del medio muro de madera, que genera disminuciones en todas las estaciones ya que es este muro el que concentra la mayor cantidad de pérdidas térmicas, estableciendo una disminución de un 5.12\% para esta estrategia en los resultados anuales, posteriormente la estrategia combinada S28 logró la disminución más efectiva de todas las simulaciones para la galería vidriada con un 5.4\% y una disminución de $2.63 \%$ referente a S13 para la zona adyacente (Zona 2 ), lo que nos señala el potencial que tiene este componente construido con los materiales y normativas actuales.

\section{CONCLUSIONES}

\section{Sobre la arquitectura y los inmigrantes del s. XIX que llegaron a Valparaíso}

Lo primero que se reconoce en la investigación, es el ingenio de los colonos y pobladores de Valparaíso, que en condiciones geográficas adversas y asechados por desastres naturales, lograron adaptar un tipo de construcción particular para la ciudad, cuyo aporte cultural y arquitectónico es el que justifica el título de patrimonio de la humanidad por la UNESCO a la ciudad de Valparaíso.

En segundo lugar, de los 85 casos de viviendas con galería catastrados que cumplen con los requerimientos de sistema constructivo Balloon y Platform Frame con relleno de adobillo, se puede ver a simple vista recorriendo la ciudad, pero se reconoce que el número es mucho mayor, confirmando la valorización y repetición sistemática de este componente ambiental en la arquitectura tradicional de Valparaíso.

La característica principal de la galería vidriada en relación a las casas tradicionales con sistema constructivo Balloon y Platform Frame, radica en que el muro interior de la galería expone el revoque de barro y los adobillos a la captación solar como un muro Trombe ampliado, este componente espacial no ocurre en otras viviendas tradicionales de la misma época en Valparaíso.

\section{Sobre el análisis a través de mediciones in situ del caso de estudio}

Se obtuvo que, la influencia del muro estudiado hace que la humedad relativa descienda en promedio un $20 \%$ en el interior de la galería, tanto para invierno y verano respecto de clima exterior, provocando efectos en los promedios de temperatura registrados, ya que, ésta sube en $4^{\circ} \mathrm{C}$ en el interior de la galería para el mes de verano e invierno. Se comprueba también que, a través de las mediciones, el fenómeno de estratificación de la temperatura del aire en la galería es inverso, en ese sentido: a menor altura la humedad relativa y la temperatura son mayores, por el contrario, a mayor altura la humedad relativa y la temperatura son menores, para los dos periodos registrados, invierno y verano. Lo anterior se explica ya que la parte baja del muro tiene mayor capacidad de captación solar en relación a la superficie vidriada.

Con respecto al período de invierno, se puede decir que el efecto que genera el muro estudiado es de estabilización del comportamiento de las curvas registradas, estas se suavizan eliminando los pick que presenta el clima exterior. Por otra parte, el porcentaje de disminución de la humedad relativa es de 15\% a 5\% menos que en verano, esto puede explicarse por las bajas temperatura del período y la capacidad del muro de absorber humedad del aire.

\section{Sobre el análisis de las simulaciones del caso de estudio (Tabla 2)}

La formulación del problema proponían estudiar el comportamiento de la galería vidriada partiendo desde la orientación, el espesor del muro Platform Frame relleno de adobillo en su composición y las proporciones del sistema constructivo. En este sentido, se comprobó que la mejor orientación para los períodos de primavera y verano es la orientación oriente (51 ${ }^{\circ}$ azimut), debido a la gran superficie vidriada que posee la galería en las orientaciones norte y oeste, cuando se ve sometida a sobrecalentamiento. Para el período de otoño la mejor orientación es norte y para invierno es poniente. Sin embargo, en el desempeño anual, la orientación que tiene menor cantidad de horas de disconfort, es la oriente. 
Al evaluar las mejoras de la galería vidriada, mediante el índice de infiltración, podemos decir que se observa un aumento considerable de los índices de humedad relativa llegando al registro máximo de $100 \%$ en el período de invierno, en verano en cambio el aumento es más leve, sin embargo, los datos muestran que las máximas alzas de las horas de disconfort son en primavera y verano, por pérdida por ventilación, a diferencia de los períodos más fríos donde se registran bajas, por lo cual, el comportamiento de la galería a mayor hermeticidad eleva su temperatura y humedad relativa, producto de la pérdida de ventilación involuntaria a causa de la infiltración, lo que ayuda en periodos fríos, como se ve en las simulaciones, ya que genera menos pérdidas, pero en periodos cálidos sino se revisan los regímenes de ventilación producirá sobrecalentamiento.

Para desarrollar estudios de simulación dinámica en edificaciones patrimoniales resulta difícil obtener datos de la literatura que apliquen como parámetros estandarizados ya que estas construcciones en su mayoría carecen de mantención y testeo de sus materiales por lo cual las validaciones deben ser manipulables en virtud de la correlación con los datos arrojados por las mediciones y observaciones in situ.

Los resultados corroboraron que la estructura de madera Platform Frame con pared de adobillo es responsable del control térmico de los espacios adyacentes, actuando como mediador entre estos dos recintos, donde el espacio de la galería vidriada es a su vez el mediador entre el exterior y el ambiente interior. Fue posible, en principio determinar que este componente arquitectónico ambiental pasivo contribuye favorablemente al confort térmico en el espacio de la galería solar y en la vivienda en general. Finalmente, las conclusiones obtenidas podrían servir para la futura rehabilitación energética de viviendas patrimoniales en Valparaíso.

\section{BIBLIOGRAFÍA}

CDT (2012) Manual de Terreno. Evaluación de Daños y Soluciones para Construcciones de Tierra Cruda. Documentos Técnicos №32, CDT, Cámara Chilena de la Construcción, Edición Juan Enrique González, ISBN: 978.956-7911-21-9.

CITEC (2014) Manual de Hermeticidad al Aire de Edificaciones. Bobadilla, Ariel et al., UBB, Concepción.

Cortés, Balbino (1885) Novísima guía de hortelano, jardinero y arbolista.

Estrada, B. (1978) 1536 - 1986: Primera jornada de historia urbana: Valparaíso y el proceso de industrialización en Chile a fines del siglo XIX, Valparaíso: Ediciones Altazor.

Harris, G. (1996) Emigración y políticas gubernamentales en Chile durante el siglo XIX, Valparaíso, p. 29. Citado en Santiago Lorenzo, G. H. (2000) Vida, costumbres y espíritu empresarial de los porteños: Valparaíso en el siglo XIX, Instituto de Historia, Facultad de Filosofía y Educación, Universidad Católica de Valparaíso, p. 17.

INN (2007) Norma Chilena NCh 853 - 2007 Acondicionamiento térmico. Envolvente térmica de edificios. Cálculo de resistencias y transmitancias térmicas.

Kostof, Spiro (2003) Historia de la Arquitectura, Madrid: Editorial Alianza.

MINVU (2018) Estándares de Construcción Sustentable para Viviendas de Chile, MINVU Tomo II. Energía.
Rodríguez, Eva (2001) Diseño del Siglo XIX para un invernadero "en la casa de la reina", AEA (Revista del Archivo Español de Arte) 294, Vol. 74, pp. 140 - 150.

Simó, Trinidad (1989) Formación del espacio burgués. Fragmentos. $\mathrm{N}^{\circ} 15-16$, pp. $98-106$.

UNESCO - ICOMOS (2014) Informe de Misión de Asesoramiento para el Sitio de Patrimonio Mundial Área Histórica de Ciudad-Puerto de Valparaíso (C 959 rev). Méndez, S. y María, L. (2013) Informe remitido al Estado por el Director del Centro de Patrimonio Mundial el 22 de enero de 2014. 